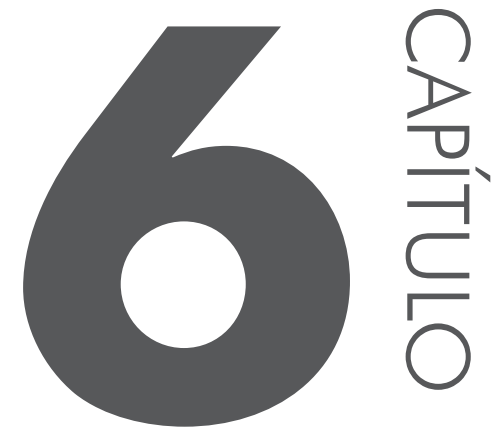

\title{
FORMALISMO HAMILTONIANO PARA A CADEIA DE TODA
}

Mateus Calixto Pereira Santos ${ }^{1}$ Paulo Eduardo Gonçalves de Assis ${ }^{1}$

Agradecimentos: Agradecemos à Fundação de Amparo à Pesquisa do Estado de Goiás (FAPEG) pelo apoio financeiro que possibilitou a realização deste trabalho.

Resumo: Neste trabalho, fazemos uma revisão sobre o modelo de Toda sob o ponto de vista hamiltoniano, visto que essa formulação é aplicável para uma vasta gama de sistemas dinâmicos, o que não é verdade para a formulação de Lax. No entanto, combinando-se elementos de ambos os formalismos, pode-se produzir uma função hamiltoniana alternativa, com um menor número de variáveis, o que pode trazer benefícios ao tratar o problema. Mostramos que a cadeia

1 Unidade Acadêmica Especial de Física e Química, Universidade Federal de Goiás - Regional Catalão, Catalão, Brasil.

E-mail de contato: mateuscalixtopereira@gmail.com, paulo.assis@ufg.br 
de partículas em estudo pode ser descrita em termos de um par de equações de Hamilton para as coordenadas canonicamente conjugadas, posição e momentum. Fazendo uso da formulação de Lax para as redes de Toda, somos capazes de reescrever o sistema por meio de uma hamiltoniana alternativa, em termos do chamado espectro auxiliar.

Palavras-chave: Cadeia de Toda. Formalismo hamiltoniano. Funções espectrais. Espectro auxiliar.

Abstract: In this work we review the so called Toda molecule from a Hamiltonian point of view, as this formula is applicable for a wide range of dynamic systems, which is not true for the Lax formulation. However, combining elements of both the formalisms can produce an alternative Hamiltonian function having a smaller number of variables, which can be beneficial when treating the problem. We show that the chain of particles under investigation can be described in terms of a pair of Hamilton's equations for the canonically conjugate variables, namely position and momentum. Making use of a previous study on the Lax formulation of the Toda model we can rewrite the system through an alternative Hamiltonian, with the help of the auxiliary spectrum.

Keywords: Toda chain. Hamiltonian formalism. Spectral functions. Auxiliary spectrum.

\section{INTRODUC̣ÃO}

O estudo de sistemas compostos de inúmeros corpos é um objeto importante para a física contemporânea, uma vez que estes podem descrever fenômenos naturais complexos. Contudo, tal estudo representa um desafio para a física e a matemática, devido à dificuldade ou até mesmo à proibição da construção de soluções exatas e simulações numéricas.

Dentre a gama de sistemas dinâmicos, há uma classe que apresenta vantagem: alguns sistemas podem ser completamente caracterizados em qualquer instante, ou seja, apresentam soluções em forma fechada. Estes modelos são denotados integráveis. Os sistemas exatamente integráveis ganharam força apenas a partir da década de 1950, mas suas primeiras observações surgiram há mais de um século, ainda que de forma incompreendida, com as ondas solitárias que aparecem em fluidos. Estas, posteriormente chamadas de sólitons, correspondem a soluções estáveis (com respeito a colisões e interações) que aparecem em sistemas não lineares, sejam eles contínuos ou discretos (TODA, 1989). 


\subsection{Redes não lineares e o problema de Fermi-Pasta-Ulam}

O problema de Fermi-Pasta-Ulam (FPU) foi um dos precursores das simulações computacionais na área da física e constitui-se de simulações em uma rede de partículas ligadas entre si através de molas que obedecem a Lei de Hooke com uma correção não linear quadrática (TODA, 1989). Esperava-se que a deformação linear quebrasse o comportamento ergódico do sistema, mas, surpreendentemente, os estados visitados durante a evolução não eram quaisquer valores. A distribuição de energia entre os modos apresentava um comportamento aproximadamente periódico, fato atribuído à presença de simetrias escondidas.

$$
\mathrm{F}=-\mathrm{k} \Delta \mathrm{x}- \pm \mathrm{k} \Delta \mathrm{x}^{2}
$$

em que $\alpha$ é o parâmetro de deformação (arbitrariamente grande ou pequeno) e $\Delta x$ é o deslocamento em relação ao ponto de equilíbrio. Logo, a força experimentada por uma partícula $j$ na rede de FPU permite-nos escrever a equação de movimento satisfeita por $\mathrm{x}_{\mathrm{j}}$ como:

$$
m \ddot{x}_{j}=k\left(x_{j+1}-2 x_{j}+x_{j-1}\right)\left[+ \pm\left(x_{j+1}-x_{j-1}\right)\right] .
$$

O problema de Fermi-Pasta-Ulam não é um sistema integrável: o número de quantidades conservadas é insuficiente para restringir univocamente sua evolução. A suficiência das simetrias em uma cadeia não linear aparece nos modelos de Toda, como veremos a seguir.

\subsection{Sistemas integráveis e os sólitons}

Quando um sistema descrito em termos de equações diferenciais não lineares apresenta um número suficientemente grande de leis de conservação, ele pode ser resolvido analiticamente; logo, dizemos que este é um sistema integrável (DAS, 1989). Como exemplo, podemos citar os sistemas hamiltonianos com n graus de liberdade, os quais, para serem sistemas exatamente integráveis, devem então possuir $\mathrm{n}$ integrais de movimento analíticas e em involução entre si. Dizemos que duas $(F, G)$ quantidades estão em involução quando o seu comutador Parênteses de Poisson é nulo.

$$
\{\mathrm{F}, \mathrm{G}\}_{\mathrm{p}}=\sum_{\mathrm{i}}^{\mathrm{n}}\left(\frac{\partial \mathrm{F}}{\partial \mathrm{q}_{\mathrm{i}}} \frac{\partial \mathrm{G}}{\partial \mathrm{p}_{\mathrm{i}}}-\frac{\partial \mathrm{G}}{\partial \mathrm{q}_{\mathrm{i}}} \frac{\partial \mathrm{F}}{\partial \mathrm{p}_{\mathrm{i}}}\right)=0
$$


Nos sistemas integráveis, podemos observar um objeto físico único: o sóliton. Os sólitons foram descritos formalmente em 1834 pelo engenheiro naval britânico John Scott Russell (RUSSELL, 1844). Russel observou um fenômeno peculiar: quando a barcaça puxada por dois cavalos nas margens do canal Edinburgh, em Glasgow, era freada repentinamente, surgia uma onda única, de forma bem definida, que seguia o curso por um longo trecho do canal sem mudar de forma e velocidade (CHALUB; ZUBELLI, 2001). Esse fenômeno foi chamado de onda solitária. Posteriormente, procurou-se modelar o comportamento descrito por Russel através de diferentes aproximações; as mais famosas são dadas pelas chamadas equações de Boussinesq (BOUSSINESQ, 1877) e de KdV (KORTEWEG; DE VRIES, 1895).

Contudo, as pesquisas entorno dos sólitons ficaram inertes até o início de 1950, quando o estudo de oscilações em redes não lineares ganhou evidência, após os trabalhos de Fermi, Pasta e Ulam no problema da partição de energia (FERMI et al., 1955). Em 1981, Morikazu Toda modificou o problema de FPU introduzindo uma interação exponencial (3.3.1) entres os elementos do reticulado, tornando-o um problema integrável (TODA, 1989).

Neste trabalho, discutiremos as propriedades das chamadas cadeias de Toda, exibindo resultados analíticos e simulações computacionais. Após introduzirmos sua formulação em termos dos pares de Lax, mostraremos que, para cada configuração inicial, existe um objeto característico, denominado curva espectral, que se conserva durante toda a evolução do sistema. A partir de tal curva, descrita pelo chamado polinômio característico, podem-se extrair informações sobre a dinâmica do sistema, como será discutido nas próximas seções. Mostraremos também que, a partir da formulação de Lax, somos capazes de reescrever uma hamiltoniana alternativa para sistema em termos do chamado espectro auxiliar.

\section{METODOLOGIA}

A pesquisa apresentada é uma revisão de literatura com a apresentação de alguns resultados gráficos. A base dessa pesquisa consiste em utilizar duas formulações diferentes (Lax e Hamilton) para o modelo de Toda, a fim de reescrever uma função hamiltoniana alternativa que, em determinados casos, apresenta vantagens sobre a função hamiltoniana usual.

Essa pesquisa foi restrita a livros, utilizando, como base fundamental, Theory of Nonlinear Lattices, de Morikazu Toda, e Classical Mechanics, de Herbert Goldstein, Charles Poole e John Safko. 


\subsection{Cadeia de Toda}

A cadeia de Toda é uma rede unidimensional constituída por $\mathrm{n}$ corpos interagentes, caracterizada pela interação que depende exponencialmente da distância entre os corpos interligados (TODA, 1989). Para descrever o movimento dos corpos interligados, utilizamos os conceitos newtonianos.

O deslocamento de cada corpo discreto na rede é dado por $\left(\mathrm{q}_{\mathrm{n}}\right)$, onde $n$ indica rótulo do objeto. Assim, temos que $\dot{q}_{n}=v_{n}$ e $\dot{q}_{n}=a_{n}$, onde $v_{n}$ é a velocidade e $a_{n}$ é a aceleração do enésimo corpo discreto da rede. Portanto, seguindo a notação newtoniana, podemos escrever as leis do movimento para o sistema, considerando a massa de cada corpo unitária como:

$$
\begin{gathered}
\dot{\mathrm{q}}_{\mathrm{n}}=\mathrm{P}_{\mathrm{n}}, \\
\dot{\mathrm{p}}_{\mathrm{n}}=\mathrm{e}^{-\left(\mathrm{q}_{\mathrm{n}}-\mathrm{q}_{\mathrm{n}-1}\right)}-\mathrm{e}^{-\left(\mathrm{q}_{\mathrm{n}+1}-\mathrm{q}_{\mathrm{n}}\right)},
\end{gathered}
$$

onde $p_{n}$ é o momento linear do enésimo corpo da rede.

\subsection{Formulação de Lax para a cadeia de Toda}

A partir das equações mostradas acima, é conveniente utilizar da formulação de Lax para reescrevermos as leis de movimento, uma vez que esta apresenta vantagens que serão mostradas adiante. Mas antes disso, para uma "abreviação" nos cálculos, introduzimos duas novas variáveis, $A_{n}$ e $B_{n}$, as quais são expressas por:

$$
\begin{gathered}
A_{n}=\frac{1}{2} e^{-\frac{\mathrm{q}_{n+1}-\mathrm{q}_{\mathrm{n}}}{2},}, \\
\mathrm{~B}_{\mathrm{n}}=\frac{1}{2} \mathrm{p}_{\mathrm{n}} .
\end{gathered}
$$

Admitindo também que o sistema estudado é periódico e constituído de $\mathrm{n}$ partículas, podemos escrever as condições de contorno como : $A_{n+N}=A_{n} \mathrm{e}$ $B_{n+N}=B_{n}$.

Portanto, podemos escrever as equações do movimento reescritas com $A_{n} \mathrm{e}$ $B_{n}$, Equações (6) e (7) em termos da equação de Lax (LAX, 1968):

$$
\frac{\mathrm{dL}}{\mathrm{dt}}=[\mathrm{M}, \mathrm{L}],
$$

onde $M$ e $L$ são os chamados pares de Lax.

Os pares de Lax podem são matrizes de dimensões $\mathrm{N} \times \mathrm{N}$, que para o caso do sistema analisado, são expressos como: 


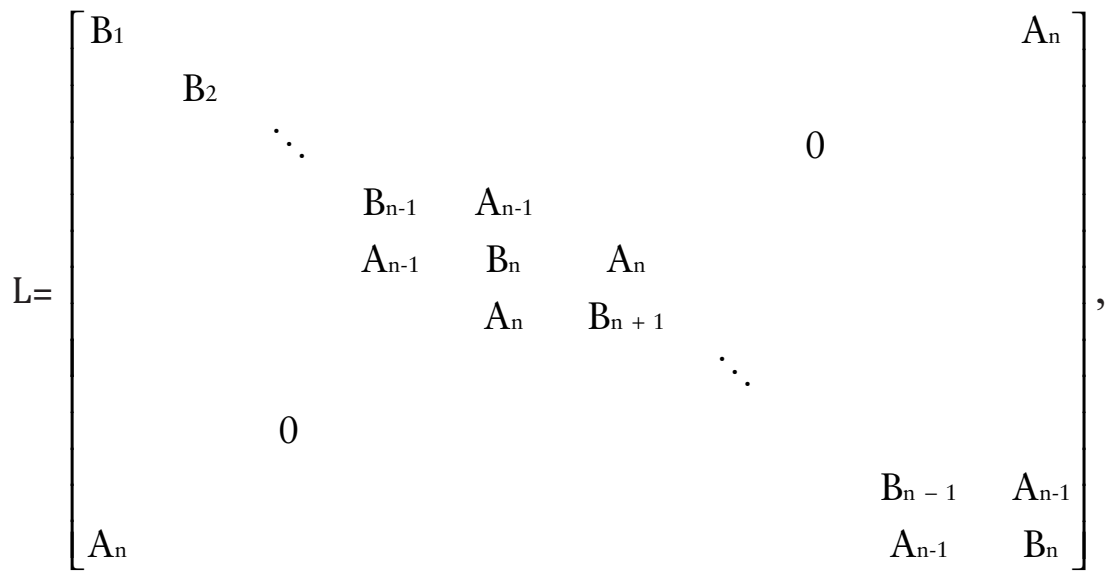

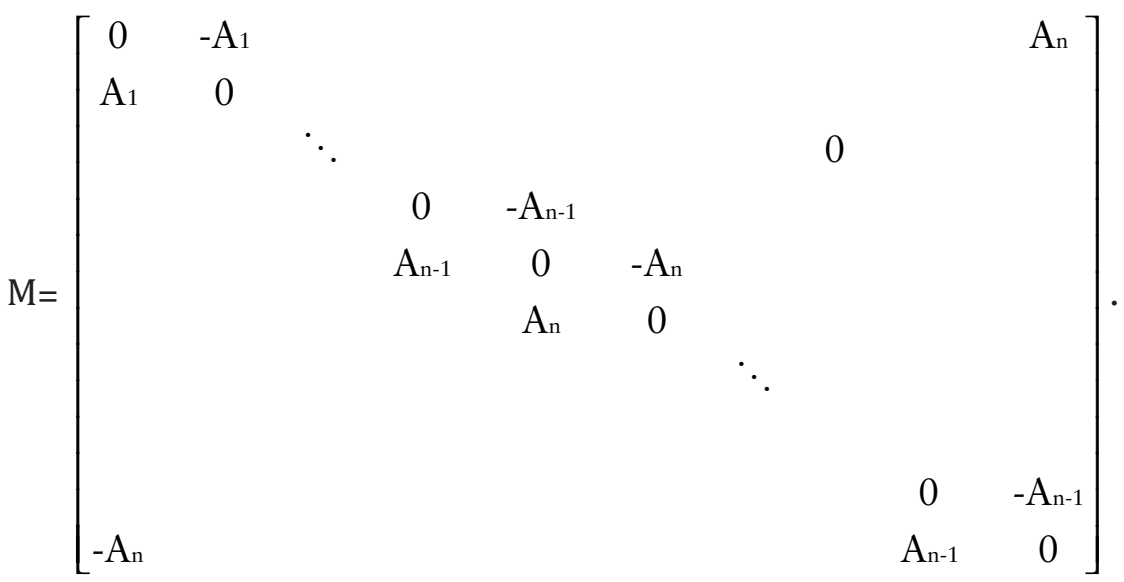

A partir da equação de Lax, para o sucesso do método, deve-se introduzir uma nova matriz $U$, que possui a função de governar a evolução da matriz $M$. Assim, podemos escrever que:

$$
\frac{d U}{d t}=M U
$$

onde esta também satisfaz as condições: $U(0)=1$ e $U U^{-1}=1$.

Além disso, é possível mostrar que a matriz $U$ também gera a evolução temporal da matriz L na forma de:

$$
L(t)=U(t) L(0) U(t)^{-1},
$$

fato este que implica, utilizando a propriedade cíclica do traço da matriz $L$, na constatação de uma lei de conservação, 


$$
\frac{d T_{r}}{d t}\left(U^{-1} L U\right)=0
$$

A Equação (11) traz um importante aspecto do traço da matriz L, em que este é invariante no tempo, característica essa que associa o traço da matriz $L$ a uma "carga" ou quantidade conservada. Outro resultado importante é que não só o traço da matriz L é conservado, mas as potências do traço também são, gerando então n quantidades conservadas no tempo, $\frac{d}{d t} T_{r}\left(L^{m}\right)=0$. Assim, podemos dizer que as $\mathrm{n}$ quantidades conservadas garantem a integralidade desse modelo, característica essa que só pode ser explicitada a partir da utilização da formulação de Lax, mostrando que esta apresenta uma vantagem significativa em relação à formulação newtoniana. Na seção de resultados, mostraremos as quantidades conservadas para o sistema com três partículas $(\mathrm{N}=3)$.

Com base nas afirmações anteriores, é mais confortável trabalharmos com autovetores e autovalores da matriz $L$; assim, podemos escrever:

$$
L(t) \varphi(t)=\lambda(t) \varphi(t) .
$$

Da evolução temporal do traço da matriz $L$, pode-se garantir que os autovalores da matriz $L$ também são invariantes no tempo; assim:

$$
\lambda(t)=\lambda(0)=\lambda,
$$

onde autovalores da matriz levam ao chamado espectro principal ou polinômio espectral.

Ainda que os $\lambda$ 's fossem conhecidos, não se poderia determinar as variáveis dinâmicas e (TODA, 1969). Logo, necessita-se de um maior número de informações sobre o sistema. Para esse propósito, define-se o espectro auxiliar $\mu$, fazendo o autovalor $\varphi_{1}(\mathrm{~N}+1)$ da matriz L igual a zero,

$$
\varphi_{1}(N+1)=0 .
$$

A Equação (14) define um valor muito especial do parâmetro espectral ( $\lambda$ $=\mu$ ), definindo o chamado espectro auxiliar $\mu_{\mathrm{i}}$, em que este, avaliado na curva espectral, fornece $\Delta\left(\mu_{\mathrm{j}}\right)$.

$\mathrm{Na}$ seção de resultados, mostraremos o espectro principal e o espectro auxiliar de forma explícita, e também faremos uma simulação numérica para o caso de $\mathrm{N}=3$.

\subsection{Formulação hamiltoniana}

O formalismo hamiltoniano é uma poderosa ferramenta matemática que permite tratar determinados tipos de sistemas físicos de forma eficiente. Pode-se 
dizer que corresponde a uma reformulação da mecânica clássica newtoniana, expressa em termos das coordenadas e dos momentos das partículas, além de, grosso modo, a energia total do sistema, uma vez que estes podem ter uma representação complexa em termos da formulação newtoniana (THORNTON; MARION, 2008).

$\mathrm{Na}$ notação newtoniana, as equações do movimento são escritas na forma de derivadas segundas no tempo, de modo que, para um conjunto de n partículas em três dimensões, tem-se um conjunto de 3 n equações diferenciais ordinárias de segunda ordem. Já o formalismo hamiltoniano baseia-se em equações de primeira ordem no tempo, porém com a desvantagem de duplicar o número de equações. Assim, no caso das n partículas, temos $6 \mathrm{n}$ equações diferenciais ordinárias de primeira ordem. Nota-se que, em ambas as situações, o número de condições iniciais necessárias é o mesmo, 6n, como seria de esperar.

As equações de Hamilton podem ser obtidas a partir de um princípio fundamental na física, o princípio de mínima ação, atribuído a Hamilton (HAMILTON, 1834; 1835), segundo o qual a trajetória seguida por um objeto durante um intervalo de tempo entre $t_{1}$ e $t_{2}$ é aquela que minimiza a ação $S$ do sistema (GOLDSTEIN, 1980),

$$
\delta S \equiv \delta \int_{\mathrm{t}_{1}}^{\mathrm{t}_{2}} \mathrm{~L}(\mathrm{q}, \dot{\mathrm{q}}, \mathrm{t}) \mathrm{dt}=0
$$

Utilizando o fato de que a função hamiltoniana é escrita em termos dos momentos lineares (p), das coordenadas (q) e do tempo (t), onde (p) e (q) são coordenadas generalizadas, além de na Lagrangeana

$$
\mathrm{L}(\mathrm{q}, \dot{\mathrm{q}}, \mathrm{t})=\mathrm{T}(\mathrm{q}, \dot{\mathrm{q}}, \mathrm{t})-\mathrm{V}(\mathrm{q}, \dot{\mathrm{q}}, \mathrm{t})
$$

substituirmos os momentos $(\mathrm{p})$ pelas velocidades $(\dot{\mathrm{q}})$ nos termos cinético $(\mathrm{T})$ e potencial $(\mathrm{V})$, e assim a integral da ação torna-se, através de uma transformação de Legandre,

$$
\delta S \equiv \delta \int_{\mathrm{t}_{1}}^{\mathrm{t}_{2}}\left\{\sum_{\mathrm{i}}^{\mathrm{N}} \mathrm{p}_{\mathrm{i}} \dot{\mathrm{q}}_{\mathrm{i}}-\mathrm{H}(\mathrm{p}, \mathrm{q}, \mathrm{t})\right\} \mathrm{dt}=0
$$

Admitindo uma variação qualquer tal que $p_{i}$ e $q_{i}$ sejam variáveis dinâmicas independentes entre si, obtêm-se a equação de Hamilton:

$$
\begin{aligned}
& \dot{\mathrm{q}}_{\mathrm{i}}=-\frac{\partial \mathrm{H}}{\partial \mathrm{p}_{\mathrm{i}}}, \\
& \dot{\mathrm{p}}_{\mathrm{i}}=-\frac{\partial \mathrm{H}}{\partial \mathrm{q}_{\mathrm{i}}} .
\end{aligned}
$$


No caso de se trabalhar no formalismo hamiltoniano, podemos escrever duas funções quaisquer $\mathrm{F}$ e $\mathrm{G}$ em termos das variáveis independentes p e q. É conveniente introduzir a operação Parênteses de Poisson (citada anteriormente) entre esses objetos:

$$
\{F, G\}_{p}=\sum_{i}^{n}\left(\frac{\partial F}{\partial q_{i}} \frac{\partial G}{\partial p_{i}}-\frac{\partial G}{\partial q_{i}} \frac{\partial F}{\partial p_{i}}\right)
$$

É fácil perceber que os Parênteses de Poisson entre as coordenadas e os momentos lineares satisfazem $\left\{\mathrm{q}_{\mathrm{i}}, \mathrm{p}_{\mathrm{j}}\right\}=\delta_{\mathrm{ij}}$.

Sempre que duas quantidades dinâmicas A e B satisfizerem uma relação como essa, elas serão chamadas de variáveis canonicamente conjugadas. Os Parênteses de Poisson possuem inúmeras utilidades, porém uma das principais é dizer se o sistema físico analisado contém simetrias, como mostraremos a seguir.

Se uma função F depende das coordenadas generalizadas (p), (q) e do tempo (t) então a taxa de variação de $\mathrm{F}(\mathrm{q}, \mathrm{p}, \mathrm{t})$ com relação ao tempo pode ser expressa como

$$
\frac{\mathrm{dF}}{\mathrm{dt}}=\frac{\partial \mathrm{F}}{\partial \mathrm{q}_{\mathrm{i}}} \frac{\partial \mathrm{q}_{\mathrm{i}}}{\partial \mathrm{t}}+\frac{\partial \mathrm{p}_{\mathrm{i}}}{\partial \mathrm{t}} \frac{\partial \mathrm{F}}{\partial \mathrm{p}_{\mathrm{i}}}+\frac{\partial \mathrm{F}}{\partial \mathrm{t}}
$$

Assim, utilizando as Equações (18) e (19), podemos obter

$$
\frac{\mathrm{dF}}{\mathrm{dt}}=\{\mathrm{F}, \mathrm{H}\}_{\mathrm{p}}+\frac{\partial \mathrm{F}}{\partial \mathrm{t}}
$$

e se a quantidade dinâmica $\mathrm{F}$ independe explicitamente do tempo, então o último termo não contribui. Pode-se então mostrar que, para que a quantidade F seja conservada ao longo da evolução, devemos ter $\{F, H\}_{p}=0$. Assim, se quisermos saber se um objeto não varia durante o movimento, é suficiente que determinemos se os Parênteses de Poisson dessa função anulam-se com respeito à hamiltoniana. Como se vê, essa operação que foi introduzida está intimamente relacionada às leis de conservação de um sistema e suas simetrias.

\section{RESULTADOS}

Considerando um sistema periódico, composto de três corpos $(\mathrm{N}=3)$, temos que a matriz L é dada por:

$$
\mathrm{L}=\left[\begin{array}{ccc}
B_{1} & A_{1} & 0 \\
A_{1} & B_{2} & A_{2} \\
0 & A_{2} & B_{3}
\end{array}\right]
$$


cujos autovalores devem satisfazer $\operatorname{det}(\lambda \mathrm{I}-\mathrm{L})=0$. Assim, obtemos um polinômio característico de grau 3, invariante e que define a chamada curva espectral do problema,

$$
\begin{gathered}
-\lambda^{3}+\left(B_{1}+B_{2}+B_{3}\right) \lambda^{2}+\left(-B_{2} B_{3}-B_{2} B_{3}-B_{1} B_{2}+A^{2}{ }_{2}+A^{2}{ }_{1}\right) \lambda^{2}+ \\
\left(B_{1} B_{2} B_{3}-A^{2}{ }_{2} B_{1}-A^{2}{ }_{2} B_{3}\right)=0 .
\end{gathered}
$$

Posteriormente, tomando o traço da matriz $\mathrm{L}$ para o caso $\mathrm{N}=3$, podemos encontrar as quantidades conservadas na forma de:

$$
\mathrm{T}_{\mathrm{r}} \mathrm{L}=\left(\mathrm{B}_{1}+\mathrm{B}_{2}+\mathrm{B}_{3}\right)=\frac{1}{2}\left(\mathrm{p}_{1}+\mathrm{p}_{2}+\mathrm{p}_{3}\right)=\frac{1}{2} \mathrm{P},
$$

onde P é o momento linear total do sistema. Outro resultado importante pode ser notado aplicando as relações de Girard, o que evidencia

$$
\mathrm{T}_{\mathrm{r}} \mathrm{L}=\left(\mathrm{B}_{1}+\mathrm{B}_{2}+\mathrm{B}_{3}\right)=\lambda_{1}+\lambda_{2}+\lambda_{3}
$$

em que $\lambda_{1}, \lambda_{2}$ e $\lambda_{3}$ são as raízes do polinômio espectral.

Assim, como dito nas seções anteriores, as potências do traço da matriz L também eram quantidades conservadas no tempo; logo, podemos encontrar:

$$
\mathrm{T}_{\mathrm{r}} \mathrm{L}^{2}=\mathrm{B}_{1}^{2}+\mathrm{B}_{2}^{2}+\mathrm{B}_{3}^{2}+2 \mathrm{~A}_{1}^{2}+2 \mathrm{~A}_{2}=\frac{1}{4} \mathrm{P}^{2}+\frac{1}{2}\left[\mathrm{e}^{-\left(\mathrm{Q}_{2}-\mathrm{Q}_{1}\right)}+\mathrm{e}^{-\left(\mathrm{Q}_{3}-\mathrm{Q}_{2}\right)}\right]=\frac{1}{2} H .
$$

Logo, pode-se verificar que, tomando o traço das potências de L relacionadas a um sistema de $\mathrm{n}$ corpos discretos, obteremos quantidades conservadas no tempo, dadas pela expressão geral:

$$
I_{k}=T_{r} L^{k}=\sum_{I=1}^{N} \lambda_{I}^{k},(\mathrm{y}=1,2,3, \ldots, \mathrm{N}) .
$$

Embora tenha sido fácil identificar as duas primeiras quantidades conservadas como sendo momento linear total e energia total do sistema, as cargas seguintes carecem de uma interpretação física intuitiva.

Existem outros sistemas assim além da cadeia de Toda, os quais, apesar de não evidenciarem as quantidades conservadas, permitem que estas sejam encontradas a partir de outros tipos de pares de Lax.

Podemos então encontrar a função hamiltoniana alternativa do sistema, utilizando primeiramente a Equação (12), e assim temos que os autovetores são dados por:

$$
\varphi_{1}(2)=-\frac{\mathrm{A}_{0}}{\mathrm{~A}_{1}}
$$




$$
\begin{gathered}
\varphi_{1}(3)=-\frac{A_{0}}{A_{1} A_{2}}\left(\lambda-B_{0}\right), \\
\varphi_{1}(4)=-\frac{\left(\lambda-B_{0}\right)\left(\lambda-B_{2}\right)}{A_{1} A_{2}}+\frac{A_{1}}{A_{2}}, \\
\varphi_{2}(2)=\frac{\left(\lambda-B_{1}\right)}{A_{1}}, \\
\varphi_{2}(3)=\frac{\left(\lambda-B_{1}\right)\left(\lambda-B_{2}\right)}{A_{1} A_{2}}-\frac{A_{1}}{A_{2}}, \\
\varphi_{2}(4)=\frac{\left(\lambda-B_{1}\right)\left(\lambda-B_{2}\right)\left(\lambda-B_{0}\right)}{A_{1} A_{2} A_{3}}-\frac{A_{2}\left(\lambda-B_{1}\right)}{A_{0} A_{1}}-\frac{A_{1}\left(\lambda-B_{0}\right)}{A_{2} A_{0}} .
\end{gathered}
$$

Sabe-se que a energia mecânica total do sistema periódico de três partículas, parametrizado em termos de e, é escrita na forma de

$$
H=2\left(\mathrm{~B}_{1}^{2}+\mathrm{B}_{2}^{2}+\mathrm{B}_{0}^{2}\right)+4\left(\mathrm{~A}_{1}^{2}+\mathrm{A}_{2}^{2}+\mathrm{A}_{0}^{2}\right)
$$

Então, podemos reexpressar a função hamiltoniana acima em termos de seu espectro auxiliar $\mu_{\mathrm{i}}$, na seguinte forma:

$$
\mathrm{H}=\frac{4\left(\mu_{1}{ }^{3}-\mu_{2}{ }^{3}\right)}{\mu_{1}-\mu_{2}}-\frac{1}{2}\left[\frac{\Delta\left(\mu_{1}\right)}{\mu_{1}-\mu_{2}}+\frac{\Delta\left(\mu_{1}\right)}{\mu_{2}-\mu_{1}}\right]+\frac{\mathrm{P}^{2}}{2}-2\left(\mu_{1}+\mu_{2}\right) \mathrm{P},
$$

utilizando as equações mostradas a seguir (TODA, 1989), onde P corresponde ao momentum total do sistema, que pode ser eliminado com uma escolha apropriada do sistema de referencial.

$$
\begin{gathered}
B_{1}+B_{2}+B_{3}=\text { constante } \equiv \frac{P}{2}, \\
\mu_{1}+\mu_{2}=B_{2}+B_{0}, \\
\mu_{2}-\mu_{1}=\sqrt{\left(B_{2}-B_{0}\right) \dagger+4 A_{2}^{2}} .
\end{gathered}
$$

É interessante notar que as novas variáveis, $\mu_{\mathrm{i}}$ e $v_{\mathrm{j}}, v_{\mathrm{j}}=\operatorname{arccosh}\left(\Delta \mu_{\mathrm{i}} / 2\right)$, satisfazem uma relação análoga à $\left\{\mathrm{q}_{\mathrm{i}}, \mathrm{p}_{\mathrm{j}}\right\}=\delta_{\mathrm{ij}}$, de modo que podem ser interpretadas como novas coordenadas canonicamente conjugadas $\left\{\mu_{\mathrm{i}}, v_{j}\right\}=\delta_{\mathrm{ij}}$. 


\section{DISCUSSÃO}

Foram discutidas nas seções anteriores algumas propriedades da cadeia de Toda. Agora, será mostrada graficamente uma excitação no modelo de Toda:

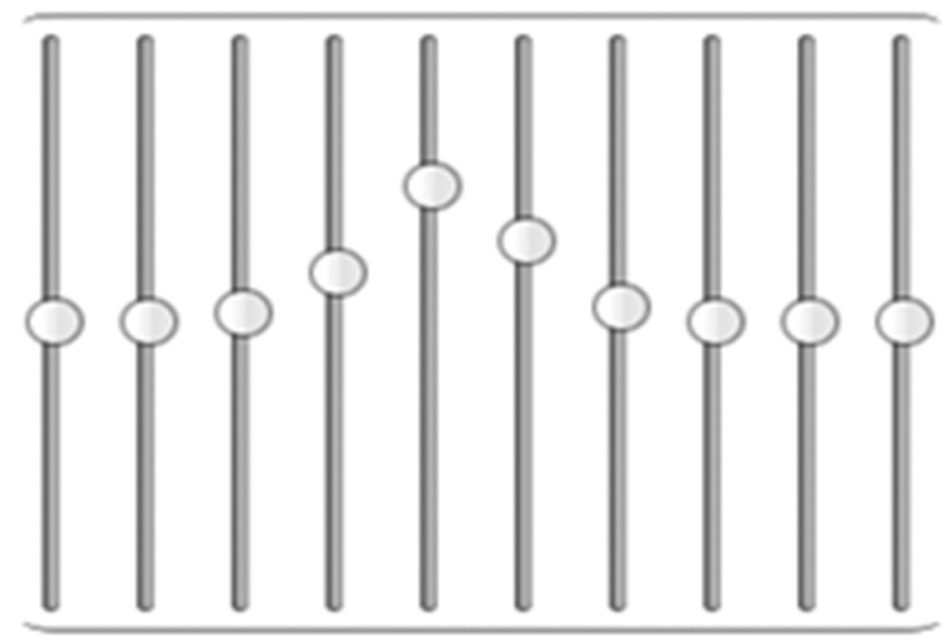

Figura 1. Representação gráfica de uma excitação localizada em uma rede de Toda com 10 partículas interagentes em um dado instante.

Na Figura 1, cada um dos corpos tem um grau de liberdade relacionado ao movimento na direção do eixo vertical e todos eles estão acoplados entre si por uma força que depende exponencialmente da distância entre as partículas. O perfil apresentado corresponde a um pulso que se propaga na rede sem dispersão ou dissipação, assim como as ondas observadas por John Scott Russell em 1843. Contudo, há uma diferença clara entre o modelo de Toda apresentado anteriormente e o sistema canal observado por Russell. A diferença se apresenta na quantidade de partículas dos dois sistemas. Conforme o número de partículas da rede cresce indefinidamente, $N \rightarrow \infty$, a rede aproxima-se do limite contínuo, e nesse regime é possível mostrar a equivalência entre a cadeia de Toda e a equação de Korteweg e De Vries (TODA, 1989). Desse modo, observando a Figura 2, podemos perceber que quando o número de partículas cresce, as trajetórias se completam, de modo a formar uma superfície ondulatória contínua, assim como os sólitons apresentados na equação de Korteweg e De Vries, mostrada na Figura 3.

$\mathrm{Na}$ Figura 2, pode-se perceber a trajetória de cada corpo por meio de uma linha contínua. Como se vê, a trajetória de cada objeto influencia a trajetória do objeto adjacente, possibilitando a propagação do pulso. 


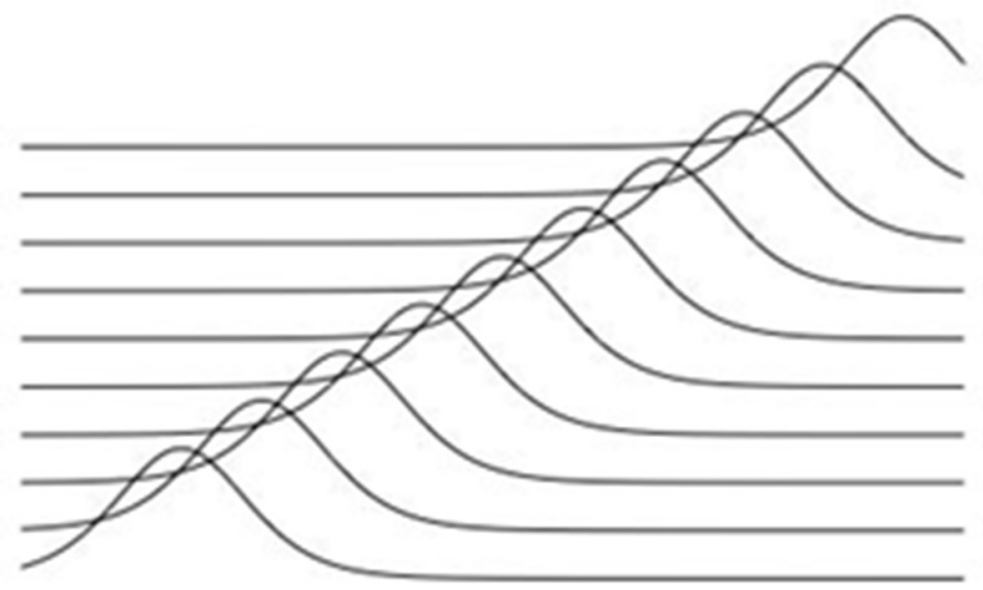

Figura 2. Evolução temporal da distensão vertical das partículas em uma cadeia de Toda com 10 corpos. No eixo vertical, está representada a posição de uma das partículas, enquanto 0 eixo horizontal está associado à coordenada temporal.

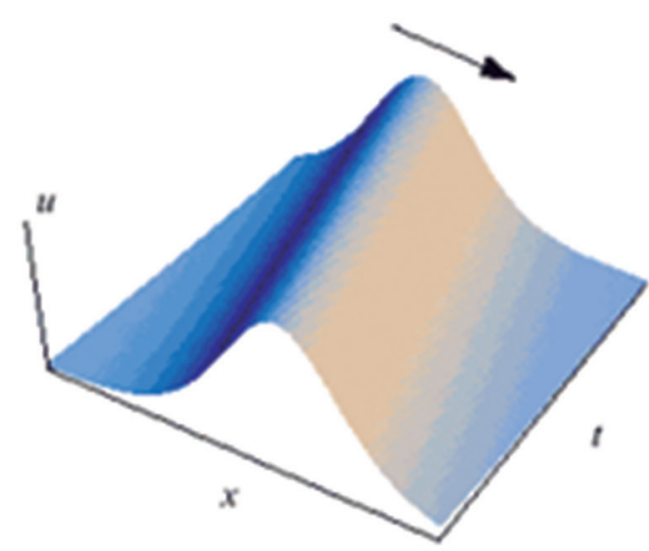

KdV I-soliton

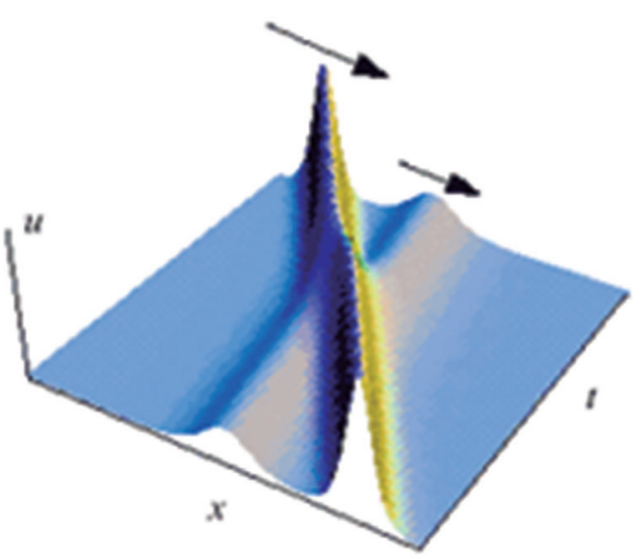

KdV 2-soliton

Figura 3. Evolução temporal da onda de KdV. À esquerda, tem-se uma onda solitária como a descrita por Scott Russell, enquanto à direita observa-se um processo de colisão (ou espalhamento) entre duas dessas ondas solitárias. Fonte: Brunelli (2000).

Pode-se também mostrar que o modelo de Toda é conservativo por meio de uma perturbação na rede cuja evolução é dada pelo potencial exponencial em cada instante de tempo. A Figura 4 representa a energia total em vários instantes de tempo, e percebe-se visualmente que não há dissipação no sistema, logo esse sistema pode ser considerado conservativo. 


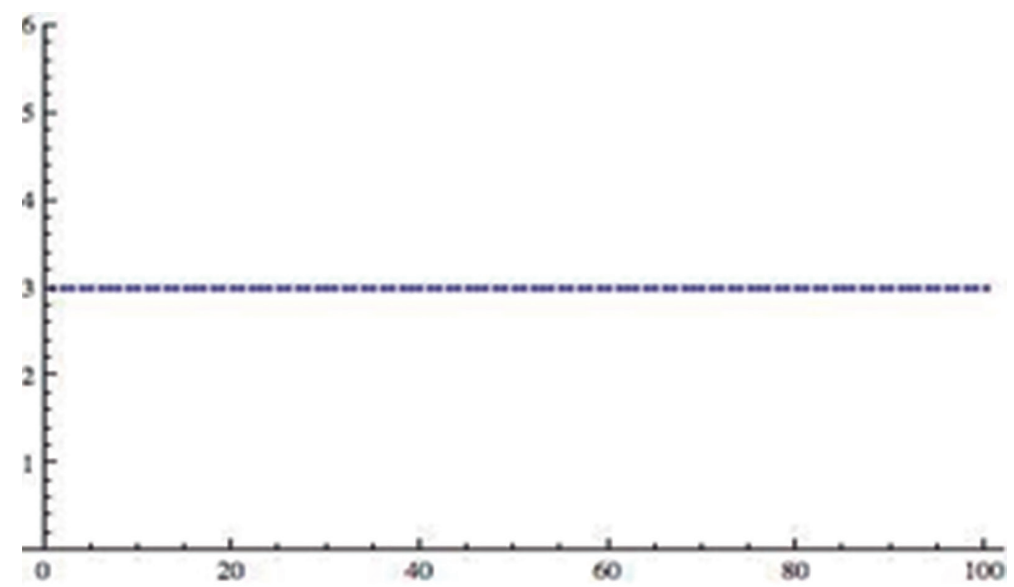

Figura 4. Enegia $\times$ Tempo para $\mathrm{N}=3$.

A cadeia de Toda é um sistema que, apesar da interação que depende exponencialmente da distância entre os corpos da rede, possui um espaço de fase familiar. Para um caso mais simples, como, por exemplo, um modelo de Toda composto de duas partículas $(\mathrm{N}=2)$, podemos escrever a energia total como:

$$
\mathrm{E}=\dot{\mathrm{x}}+\mathrm{e}^{2 \mathrm{x}}+\mathrm{e}^{-2 \mathrm{x}}
$$

onde o deslocamento da partícula 1 e 2 é dado por $q_{1}=x$ e $q_{2}=-x$.

A partir da Equação (38), podemos mostrar que o espaço de fase do modelo de Toda N=2 é dado pela Figura 5. Nela, vemos que as órbitas no espaço de fase são curvas fechadas, sendo que, para cada valor de energia, existe uma curva diferente. Quanto maior a energia, maior o comprimento da curva descrita no espaço de fase, indicando que, conforme aumenta a energia fornecida, maior é o deslocamento máximo das partículas.

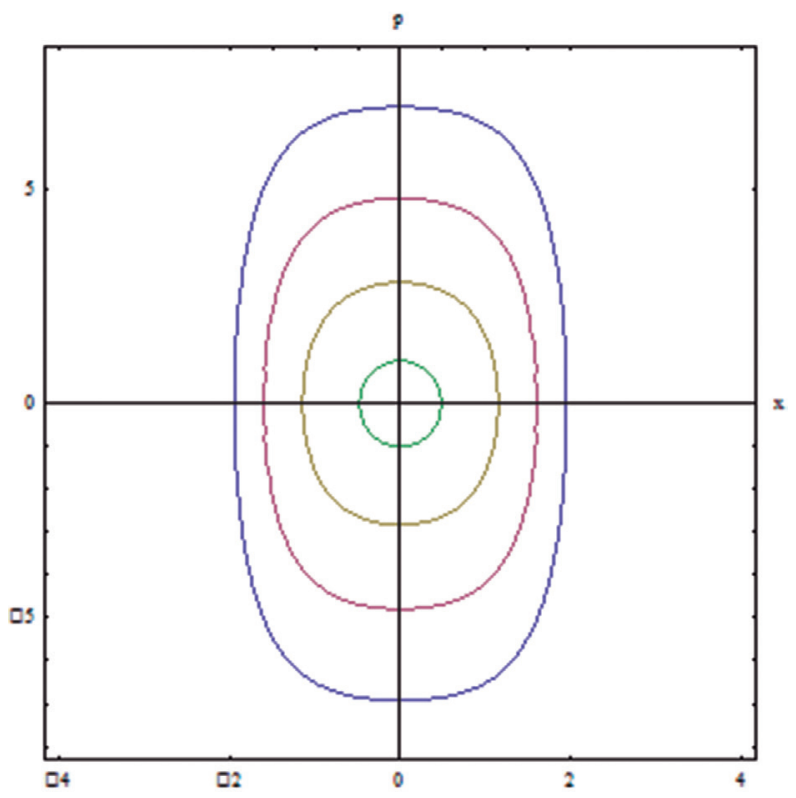

Figura 5. Espaço de fase para o modelo de Toda constituído de duas partículas. 
Analogamente, analisamos o espaço de fase de um oscilador harmônico simples unidimensional cuja equação é dada por

$$
\frac{\dot{x}^{2}}{2 E / m}+\frac{x^{2}}{2 E / k}=1
$$

e cuja representação gráfica é encontrada na Figura 6. É claro perceber que se trata de um conjunto de elipses cujos eixos crescem com a energia.

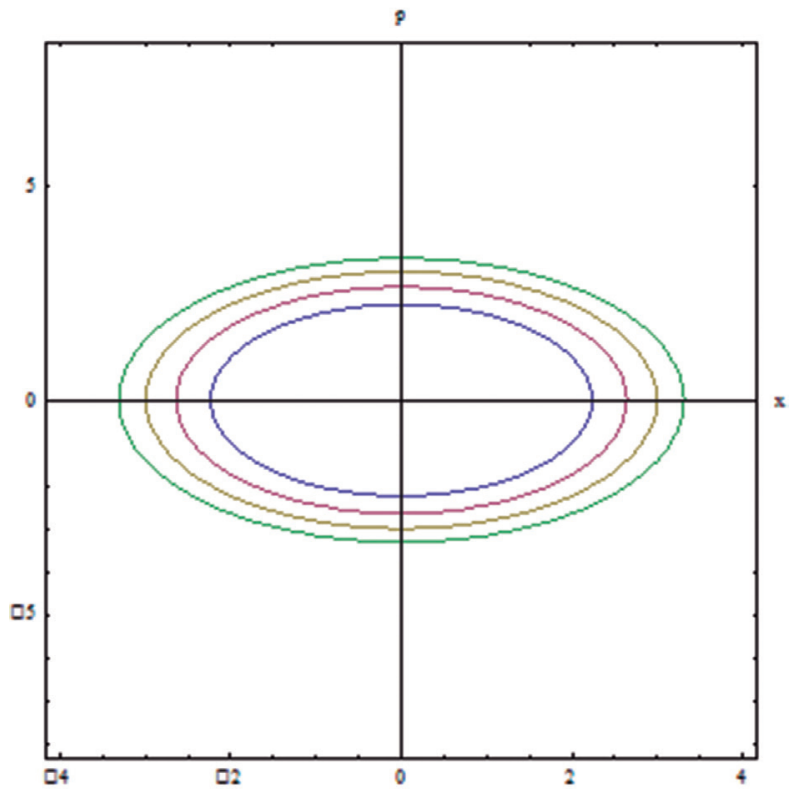

Figura 6. Espaço de fase do oscilador harmônico simples.

Analisando a Figura 5 e a Figura 6, é fácil perceber as semelhanças entre os dois sistemas e é possível pontuar as igualdades nas órbitas fechadas, apesar de esses sistemas serem dotados de leis de formação completamente diferentes um do outro.

\section{CONCLUSÃO}

Apesar de os sistemas dinâmicos constituídos de muitas partículas serem sistemas complexos de difícil tratamento - analítico ou numérico - os problemas de muitos corpos podem apresentar suficientes simetrias que lhes confiram características especiais, como a integralidade e as cargas conservadas no tempo.

Neste trabalho, mostramos que a cadeia de Toda, um sistema cujas interações internas são do tipo não lineares, admite uma formulação em termos das equações de Hamilton para as coordenadas (q) e momentos lineares (p). 
Discutimos sobre a capacidade da cadeia de Toda admitir excitações estáveis e localizadas com a presença de ondas solitárias, assim como na equação de Korteweg e De Vries (KdV).

Vimos também como a formulação de Lax para o modelo de Toda evidencia as chamadas quantidades conservadas e pode fornecer uma função hamiltoniana alternativa para ele. O espectro característico do problema permite-nos determinar o chamado espectro auxiliar com o qual foram construídas novas coordenadas canonicamente conjugadas. Tais objetos foram explicitamente construídos para um dos casos mais simples, com uma cadeia de três partículas.

\section{REFERÊNCIAS}

DAS, A. Integrable models. Cingapura: World Scientific Publishing, 1989.

BRUNELLI, J. C. Dispersionless limit of integrable models. Braz. J. Phys., v. 30, n. 2, 2000 .

BOUSSINESQ, J. Essai sur la theorie des eaux courantes. Paris: Imprimerie Nationale, 1877.

CHALUB, F. A. C. C.; ZUBELLI, J. P. Sólitons: na crista da onda por mais de 100 anos. Matemática Universitária, n. 30, p. 41-65, 2001.

FERMI, E.; PASTA, J.; ULAM, S. Studies of nonlinear problems. Document LA-1940, 1955.

GOLDSTEIN, H.; POOLE, C.; SAFKO, J. Classical mechanics. 3. ed. Addison-Wesley, 1980 .

HAMILTON, W. R. On a general method in dynamics. Philosophical Transaction of the Royal Society, 1834 and 1835.

KORTEWEG, D. J.; de VRIES, G. On the change of form of long waves advancing in a rectangular canal, and on a new type of long stationary waves. Philosophical Magazine, v. 39, p. 422-443, 1895.

LAX, P. Integrals of nonlinear equations of evolution and solitary waves. Comm. Pure Applied Math, v. 21, p. 467-490, 1968.

RUSSELL, J. S. Report on waves. Londres: John Murray, 1844.

THORNTON, S. T.; MARION, J. B. Classical Dynamics of Particles and Systems.

Boston: Brooks/Cole Cengage Learning, 2008.

TODA, M. Theory of Nonlinear Lattices. Berlim: Springer-Verlag, 1989. 